\title{
Research on Chaotic Scrambling Colourful Digital Watermarking Technology Based on DT-CWT
}

\author{
CUI Ruibo ${ }^{1}$, WANG Wenju ${ }^{1+}$, SHEN Lijun ${ }^{1}$,LI Jinming ${ }^{1}$, TANG Lijing ${ }^{1}$, Liu Jiayin ${ }^{2}$ \\ ${ }^{1}$ Shanghai University of Science and Technology, Shanghai 200093 \\ ${ }^{2}$ The Information Center of Shandong Province Tobacco Company, Jinan 250101
}

\begin{abstract}
In order to improve the invisibility of watermark information, this paper proposes a technique of embedding watermark according to the direction of digital colorful image texture. First, the scrambling watermarking Image is obtained by chaotic scrambling and Fast Fourier Transformation (FFT). Second, the embedding channel of the colorful carrier image is chosen which depends on human visual characteristics and PSNR, and Gray Level Co-occurrence Matrix is applied to judge the direction which is suitable for the Dual-Tree Complex Wavelet Transformation (DTCWT) decomposition directions $\left( \pm 15^{\circ}, \pm 45^{\circ}, \pm 75^{\circ}\right)$.Third, the chosen channel is DTCWT decomposed into the third level, the sub-image which is suitable for the direction is substituted by the scrambling watermarking Image. In the end, the decomposed carrier image is inverse Dual-Tree Complex Wavelet transformed to obtain the watermarked image. In the extraction procession, the conjugate image generated by FFT is corrected by inverse chaotic scrambling. Results: Experimental results show that the algorithm can well embed the watermark into the colorful image, and the invisibility of the watermarking image is better in the image area with the texture direction.
\end{abstract}

Keywords: blind watermarking, DTCWT, digital watermarking, FFT, Chaotic scrambling.

\section{Introduction}

In recent years, digital watermarking technology has been widely used in copyright identification and product counterfeiting for its good encryption. There are two major types of transformations commonly used by researchers today: one is FFT of the carrier image with embedding of the watermark in the transform domain [1]. The other one is the DWT of watermarking. Above mentioned methods have a small impact on the carrier image, but the watermarking is flimsy and does not have good identification [2].

The watermarking technique based on DTCWT proposed in this paper overcomes the lack of directional selectivity of DWT. The embedding direction of watermark can be adaptively selected according to the direction of image texture, and the optimal watermark embedding channel is selected to improve the visual invisibility of watermark information.

\section{Knowledge Background}

\subsection{Dual-Tree complex wavelet transform}

DTCWT is proposed by Kingsbury etc. [3] to improve DWT. It has good directional selectivity, translation invariance, complete reconstruction, data redundancy, better anti-compression, noise robust and the robustness of the attack.

The dual-tree complex wavelet is transformed by two binary tree structures to generate transformed real and imaginary parts. Two low frequency subgraphs and six complex high frequency subgraphs $\left( \pm 15^{\circ}, \pm 45^{\circ}, \pm 75^{\circ}\right)$ are generated in each level decomposition (Fig. 2). The Q-Shift [4] transformation

Corresponding author. Tel.: + 13636675266 .

E-mail address:wangwenju666@163.com. 
proposed by Kingsbury enables a more subtle implementation of the half-sample period delay between two tree filters.

Promoted by one-dimensional dual-tree complex wavelet:

$$
\begin{aligned}
& \psi(x, y)=\psi(x) \psi(y)=\left[\psi_{h}(x)+j \psi_{g}(y)\right]\left[\psi_{h}(y)+j \psi_{g}(y)\right]= \\
& \psi_{h}(x) \psi_{h}(y)-\psi_{g}(x) \psi_{g}(y)+j\left[\psi_{g}(x) \psi_{h}(y)+\psi_{h}(x) \psi_{g}(y)\right]
\end{aligned}
$$

dual-tree real wavelet:

dual-tree complex wavelet:

$$
\psi_{h}(x) \psi_{h}(y)-\psi_{g}(x) \psi_{g}(y)
$$

$$
\psi_{g}(x) \psi_{h}(y)+\psi_{h}(x) \psi_{g}(y)
$$

DTCWT decomposition process: Firstly, line transform is done by $\left\{h_{0}(n), h_{1}(n)\right\}$ to the image, and then use the filter $\left\{g_{0}(n), g_{1}(n)\right\}$ column transformation to get a low-frequency sub-band and three high-frequency sub-band; moreover, use $\left\{g_{0}(n), g_{1}(n)\right\}$ to do line transform, and use the filter $\left\{h_{0}(n), h_{1}(n)\right\}$ to do column transform to get a low frequency sub-band and three high frequency sub-bands; the sum or difference of each pair of sub-bands constitutes two low frequency coefficients and six high frequency coefficients. Fig.1.

$\begin{array}{llll}\text { low-pass L: } & A_{1}+A_{2} & A_{1}-A_{2} & \\ \text { high-pass B }\left({ }_{-}^{ \pm} 75^{\circ}\right): & H_{1}+H_{2} & H_{1}-H_{2} \\ \text { high-pass B }\left({ }_{ \pm}^{+} 15^{\circ}\right): & V_{1}+V_{2} & V_{1}-V_{2} \\ \text { high-pass B }\left({ }^{+} 45^{\circ}\right): & D_{1}+D_{2} & D_{1}-D_{2}\end{array}$
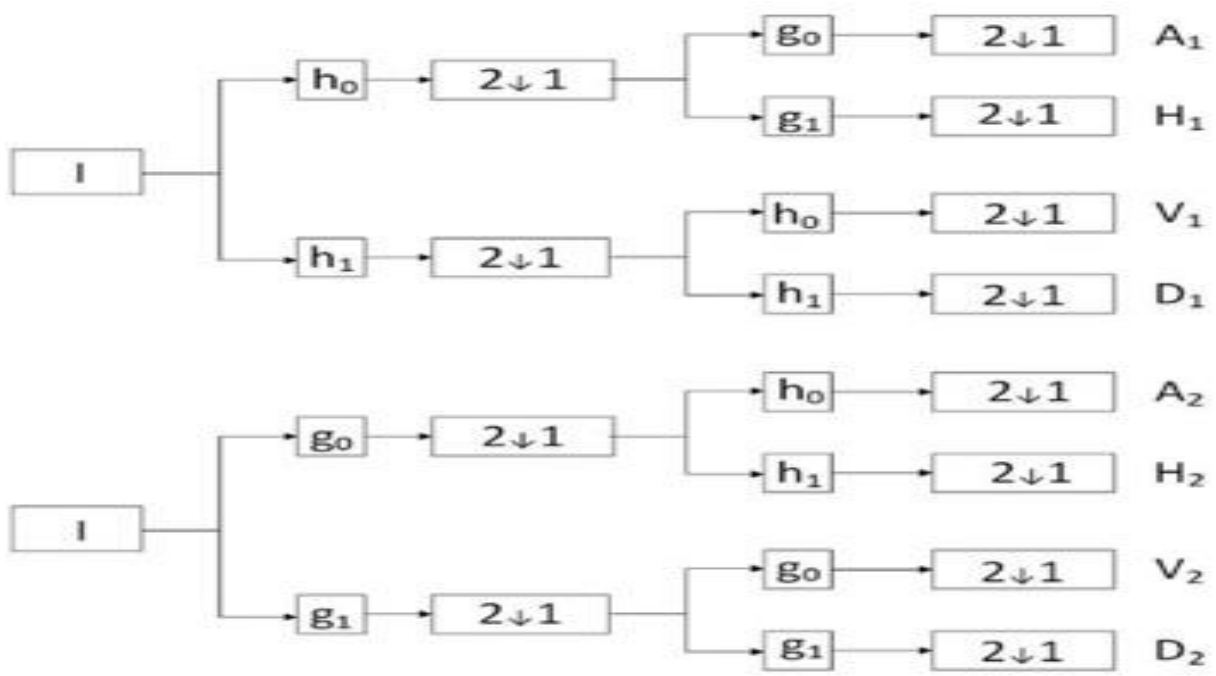

Fig. 1: wavelet decomposition map

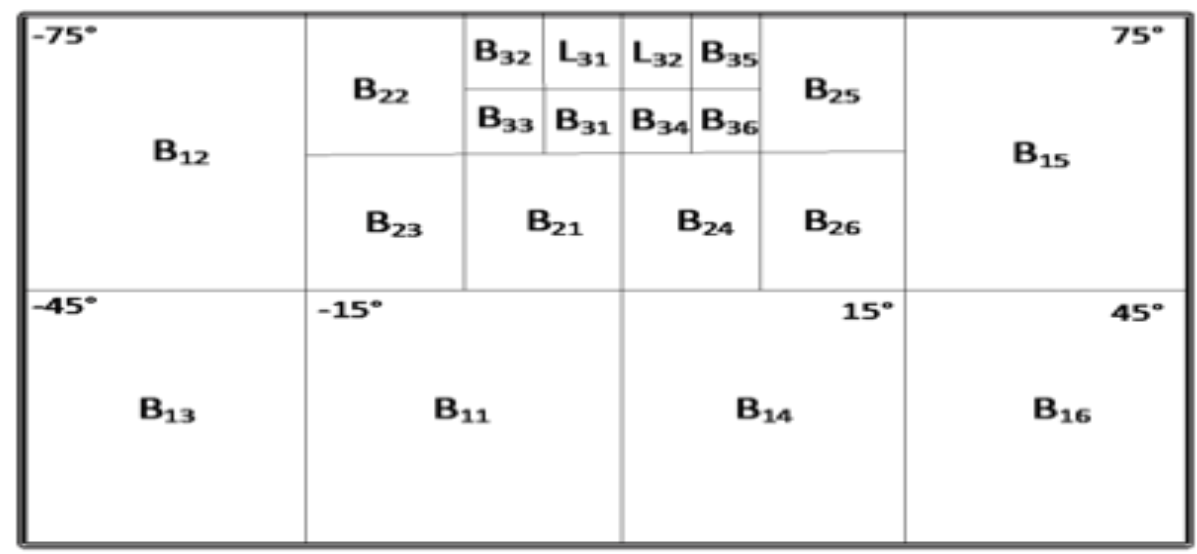

Fig. 2: Dual-tree complex wavelet decomposition 


\section{Chaotic Scramble Colorful Image Digital Watermarking Algorithm}

\subsection{Watermark embedding algorithm}

The process shown in Fig. 3.

1. According to the size of the binary watermark image, a chaotic sequence [5] of length $m \times n$ is obtained from the iterative equation

$$
x_{1}=k e y, \quad x_{n+1}=L \cdot x_{n}\left(1-x_{n}\right)
$$

2. Scramble the original watermark image $f(x, y)$ by the chaos sequence to get the chaotic image $f^{\prime}(x, y)$.

3. The scrambled image after Eq. 4 transform to obtain the frequency domain image $F(\mu, v)$.

4. The following methods are used to select the embedding channel and embedding decomposition direction:

(1) Selection of carrier image embedding channels: For each channel of the carrier image, the std value is calculated according to Eq. 6

$$
s t d=\sqrt{\frac{1}{M N} \sum_{i=1}^{M} \sum_{j=1}^{N}\left[a_{i j}-\hat{a}\right]^{2}}
$$

In Eq.6, M, N is the size of the carrier image matrix, $a_{i j}$ is the original value of the grey level of each pixel, and $\hat{a}$ is the average of the grey. The channel with smallest std value is selected.

(2) The choice of embedding direction:

Grey level co-occurrence matrix [6] for all pixels in the image conducts a survey to describe the grey distribution.

$f(x, y)$ is a two-dimensional digital image with a size of $M \times N$ and a grayscale of $N_{g}$, the grey level co-occurrence matrix satisfying certain spatial relationship is:

$$
p(i, j)=\#\left\{\left(x_{1}, y_{1}\right),\left(x_{2}, y_{2}\right) \in M \times N \mid f\left(x_{1}, y_{1}\right)=i, f\left(x_{2}, y_{2}\right)=j\right\}
$$

Which represents the number of occurrences of a pixel pair from the point of grey level " $\mathrm{i}$ ” to the point of grey level " $\mathrm{j}$ " that satisfies the relationship of the fixed position $(d, \theta) . \quad \#\{\}$ represents the number of elements in the set satisfying the formula conditions, $\left(x_{1}, y_{1}\right)$ and $\left(x_{2}, y_{2}\right)$ represent the position of the pixel, $\mathrm{d}$ represents the distance between pixels, and $\theta$ represents the angle between pixels. According to each decomposition of dual-tree complex wavelet, there are 6 complex coefficients high-frequency subgraph corresponding to 6 angles, $\theta \in\left\{ \pm 15^{\circ}, \pm 45^{\circ}, \pm 75^{\circ}\right\}$. For a certain value of $d$, for each $\theta$, the carrier images are respectively analysed according to Eq.7 to obtain 6 grey level co-occurrence matrices $p(i, j)$. For the six grey level co-occurrence matrices of the vector image, the energy $A S M$ of the grayscale matrix is calculated by Eq.8.

$$
A S M=\sum_{i=1}^{M} \sum_{j=1}^{N}(p(i, j))^{2}
$$

The $\theta$ which correspond to largest $A S M$ among the six grey matrix energies is selected。

5.According to the direction of embedding, the DTCWT decomposed subgraph $\mathrm{B}_{3 \mathrm{i}}$ is selected properly (see Fig.2). " 3 " represents the algorithm uses three-level decomposition, " $\mathrm{i}$ " represents the decomposition direction, $i=1,2,3,4,5,6$ correspond to $-15^{\circ},-75^{\circ},-45^{\circ}, 15^{\circ}, 75^{\circ}, 45^{\circ}$ respectively.

\subsection{Watermark extracting algorithm}

The process shown in Fig. 4

5. Check the colourful image to be detected.

6. According to the channel selection method and direction selection method (corresponding to step 4 (1), (2) of the embedding method respectively), the watermark embedding channel and watermark embedding direction are judged again, and the image is decomposed into three levels by DTCWT. 
7. Through IFFT, the watermarked frequency domain image is turned to Scrambled image. Then applying chaotic decryption to the scrambled image to obtain the watermarking image.

8. The watermarking image is processed with de-noising by median filtering and other operations to obtain a better quality. This method does not require the participation of the original watermark image and belongs to the blind watermark extraction technique.

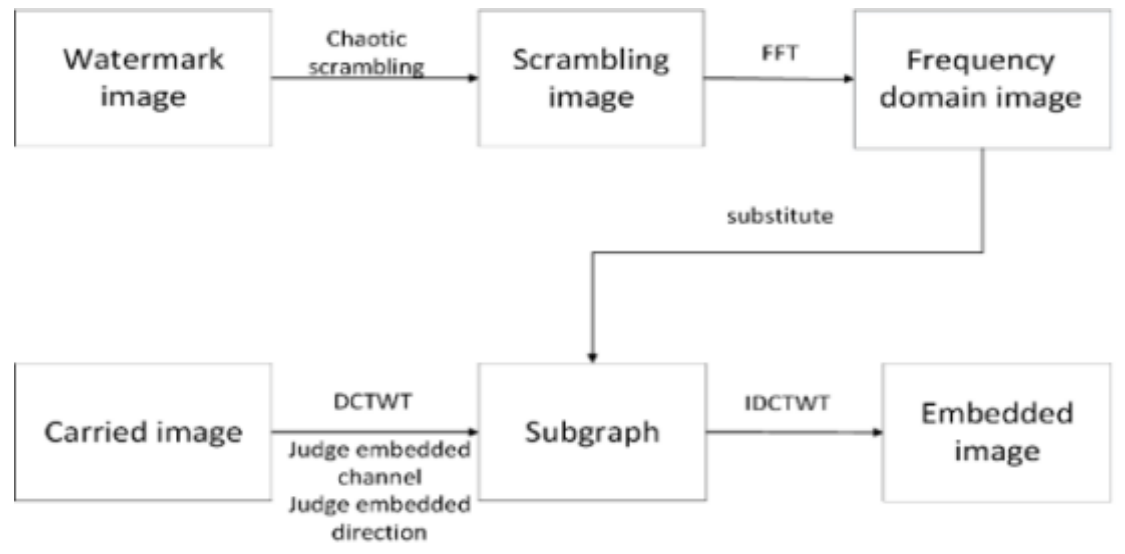

Fig. 3: Watermark embedding flow chart

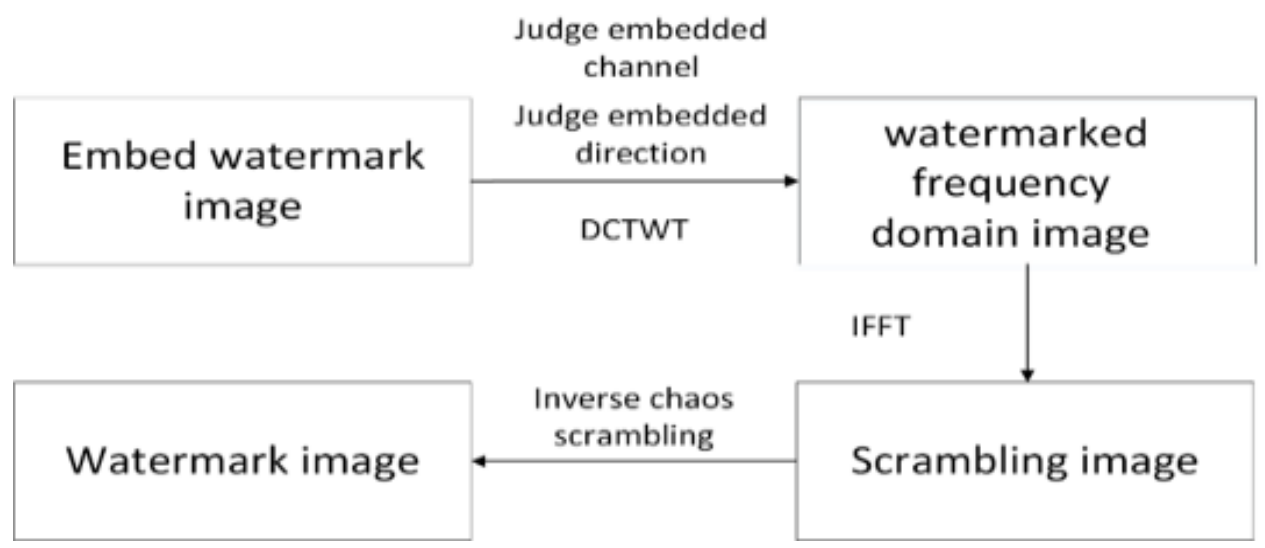

Fig.4: Watermark extraction flow chart

\section{Experimental Results and Analysis}

\subsection{Algorithm results and analysis}

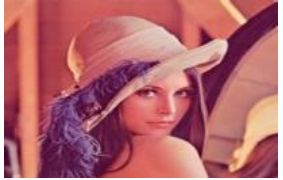

Fig. 5

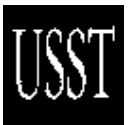

Fig. 6

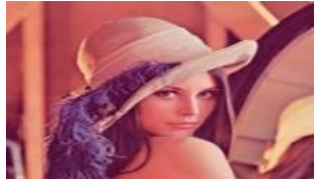

Fig. 7

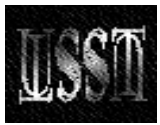

Fig. 8

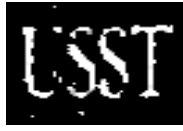

Fig .9

\subsubsection{Invisibility test}

Fig.5 shows the original carrier image, according to the embedding method, the DTCWT coefficient B33 is selected.

Fig.7 is the image with embedding intensity $K=20$. Although the PSNR [7] is 27.1613, it has little effect on the human visual system. Fig. 8 shows the watermarking without chaos scrambling. Fig.9 (NC = 0.8323 ) shows the extracted image after using chaotic scrambling, it can be seen that the chaotic scrambling eliminates the conjugate virtual image.

\subsubsection{Robustness test}


Table 1: Results of robustness test

\begin{tabular}{ccccc}
\hline attack & Salt\&Pepper(0.3) & Gaussian(0.8,0.2) & Rotation45 & Croop75\% \\
& & & & 0.7990 \\
\hline $\mathrm{NC}$ & 0.8646 & 0.8667 & 0.8326 & 0.76 \\
\hline
\end{tabular}

Table 1 shows the attack result, because of the DTCWT with good translational invariance, the watermarked image is still reconstructed clearly. This demonstrates the anti-clipping ability of the watermarking algorithm.

\section{Conclusion}

The technique proposed in this paper can eliminate the rotational symmetry caused by Fourier transformation and improve the visual effect effectively. And the watermarking can be selectively embedded in the six directions decomposed by DTCWT to improve the invisibility.

\section{Acknowledgements}

This work was supported in part by a grant from Laboratory of Green Platemaking and Standardization for Flexography Printing (ZBKT201710), the Innovation and Entrepreneurship Project of College Student of University of Shanghai for Science and Technology (No.XJ2017238, XJ10252292, XJ10252296, XJ10252297) and the Innovation and Entrepreneurship Project of College Student of Shanghai (SH10252168, SH017163).

\section{References}

[1] Jain, Reema.; Kumar, Mahendra; Jain, Arihant Kumar; et al. Digital Image Watermarking using Hybrid DWT-FFT technique with different attacks. Source: 2015 International Conference on Communication and Signal Processing, ICCSP 2015, pp 672-675.

[2] Rana, Azmat; Pareek, N.K. Comparative study of DCT and DWT techniques of digital image watermarking .Source: Smart Innovation, Systems and Technologies, ICTIS 2017, v 84, p 377-382.

[3] Kingsbury, Nick.The dual-Tree complex wavelet transform a new efficient tool for image restoration and enhancement Source: European Signal Processing Conference, 1998,v 1998-January.

[4] Kingsbury N G.Complex wavelets for shift invariant analysis and filtering of signals. Source: Applied and Computational Harmonic Analysis, 2001, v 10, n 3, p 234-253.

[5] Ahmad, Musheer; AlSharari, Hamed D. On the security of chaos-based watermarking scheme for secure communication. Source: Advances in Intelligent Systems and Computing, 2017, v 515, p 313-321.

[6] Srivastava, Prashant (Department of Electronics and Communication, University of Allahabad, School of Information and Communications, Allahabad, Uttar Pradesh, India); Khare, Manish; Khare, Ashish. Content-based image retrieval using scale invariant feature transform and gray level co-occurrence matrix. Source: Proceedings of SPIE - The International Society for Optical Engineering, 2017, v 10443.

[7] Yadav, Bandana; Kumar, Ashish; Kumar, Yogendera. A Robust Digital Image Watermarking Algorithm Using DWT and SVD.Source: Advances in Intelligent Systems and Computing, 2018, v 583, p 25-36. 